he is deluded. A phenomenological approach to psychiatry examines the experiences of the "person", but has nothing to say regarding how one marries these to scientific observations of the "organism".

There is a (probably apocryphal) story of a 17th century debate ending with the realist challenging the idealist to stop eating and drinking substances in the outside world which he did not believe existed. In the same way, an appeal to common-sense realism finishes the argument without really furthering it.

1 Lynton Road

MARK MORRIS

London N6 6BD

\section{Bulgarian Psychiatric Association}

DEAR SIRS

The Constituent Assembly of the new Bulgarian Psychiatric Association (BPA) took place in May. The BPA is an independent non-profit-making professional organisation which is registered under Bulgarian law with its head office in Sofia.

The programme adopted as a priority for its first year a critical review of the current state of psychiatry in Bulgaria, with an emphasis on human rights issues. This is perceived as essential to recover the integrity and values of the profession following their abuse under the previous political regime. In undertaking this task, the BPA will clearly benefit from experience and technical assistance from other national or international associations, especially regarding the following issues.

(a) Sensitisation, dissemination of knowledge and technical expertise leading to "good practices" in the professional training and development of psychiatrists.

(b) Reconciling the individual liberties and rights of mental patients with unhindered access to treatment when needed (for example, many services at present avoid dealing with psychiatric emergencies for fear of allegations of psychiatric abuse).

(c) Development of an appropriate and valid procedure for investigating complaints and claims of psychiatric abuse or human rights violations.

We do hope that the British psychiatric community will extend its cooperation to the BPA and assist us in attaining the goals we have set for ourselves in this first year of the organisation's existence.

Tома TомоV

President-Elect

Bulgarian Psychiatric Association

15 D. Nestorov Str.,

1431 Sofia, Bulgaria

\section{East European Medical Journal}

\section{DeAr Sirs}

Starting in August 1992, we intend to establish a new medical journal East European Medical Journal, conceived as a peer-reviewed scientific publication focusing on specific medical aspects in East and Central Europe and to be published in English.

We feel that the unidirectional flow of the best scientific papers towards Western journals represents a danger for local cultural experience. One of the keys to educational, social and cultural development could be high quality journals that create opportunities for self expression of local scientists and for developing a sense of regional unity and historic tradition. These publications could play a significant role in pushing people to compete and creating new leaders. Creating a new elitism in our society and supporting it by a scientific culture becomes an important social claim.

It is about this development project that I approach you for help. We need to involve some Western colleagues in order to provide assistance in the editorial processing of manuscripts and we are looking for a large team of peer reviewers. Papers addressed to this journal will be welcome.

We thank you in advance and hope to receive comments and suggestions from your readers.

TRAIAN MihXESCU

East European Medical Journal

Editor

Eurocip, P.O. Box 209

Iasi 6600

Romania

\section{Visual arts in clinical settings}

\section{DeAr Sirs}

The visual arts are used or promoted by many professions within the health and social services. Perhaps some of your readers may be interested in contributing to a conference which aims to bring these professions together in order to present and discuss the different uses and understandings of the visual arts in clinical settings. More information can be obtained by ringing Pat Coopey on Gloucester (0452) 617033 extension 2359.

TED QuinN

Head Art Therapist

(Visual Arts Conference Co-ordinator)

Gloucestershire Health Authority

Coney Hill Hospital

Gloucester GL4 7QJ 